\title{
Study on Large Area Photomultipliers with Super Bialkali Photocathode
}

\section{Emanuele Leonora ${ }^{1}$}

INFN - sezione di Catania

\section{Sebastiano Aiello}

INFN - sezione di Catania

\section{Domenico Lo Presti}

INFN - sezione di Catania, Dipartimento di Fisica ed Astronomia di Catania

\section{Valentina Giordano}

INFN - sezione di Catania

\section{Fabio Longhitano}

INFN - sezione di Catania

\section{Cristina Pugliatti}

INFN - sezione di Catania, Dipartimento di Fisica ed Astronomia di Catania

\section{Nunzio Randazzo}

INFN - sezione di Catania

The quantum efficiency of standard bialkali photocathode is currently limited to around $25 \%$ at the spectral peak. Many photomultipliers manufactures have recently developed large area photomultipliers with new super bialkali photocathode which improves quantum efficiency up to $35 \%$. In this paper the characterization of large area photomultipliers with super bialkali photocathode produced by Hamamatsu is reported, to compare their performances to those measured on standard bialkali R7081 PMTs with the same mechanical design. Time and charge characteristics, fraction of spurious pulses and dark count, uniformity and ageing effects were measured in order to define whether the new kind of photocathode could introduce some secondary effects.

International Workshop on New Photon-detectors,

Speaker: Emanuele Leonora. INFN - sezione di Catania . Via S.Sofia, 64, 95123, Catania, Italy. E-mail: emanuele.leonora@ct.infn.it 


\section{Introduction}

Large area photomultipliers (PMT) are widely used in astroparticle and neutrino experiments that need large detection area and high sensitivity to photons [1] [2]. The sensitivity is described in terms of quantum efficiency (QE), i.e. the number of photoelectrons emitted from the photocathode divided by the number of incident photons [3].

The quantum efficiency of standard bialkali photocathode is currently limited to around $25 \%$ at the spectral peak. Many photomultipliers manufactures have recently developed a new super bialkali (SBA) photocathode which improves spectral response, increasing the quantum efficiency up to $35 \%$. This paper describes in details an intense work of measurements conducted to define the overall characteristics of large area photomultipliers produced by Hamamatsu [4] with SBA photocathode of 10-inch diameter, and 10-dynodes stage, and compared to those measured on a widely used type of a 10-inch standard bialkali photocathode photomultipliers, the R7081 Hamamatsu PMT. From the point of view of the design, the two models differ only for the photocathode QE. In the case of SBA PMT, the higher QE is achieved through the use of a more refined technology used for the realization of the photocathode.

With that in mind, comparative measurements of their detection efficiency has been made, time and charge characteristics, as well as fraction of spurious pulses and dark count rate were measured, in order to define whether the new kind of SBA photocathode could introduce some secondary effects. The uniformity of the SBA photocathode was also measured and compared to that of the standard PMTs by scanning the photocathode surface with a collimated laser beam. Moreover, an accurate study about the ageing effects has been performed for a time of over three years for both the two types of PMTs.

\section{The overall characteristics measurements}

The following section report on the procedures and results of the characterization of a batch of four photomultipliers with 10-inch SBA photocathode and 10-stages produced by Hamamatsu [4]. Their performances, were compared to measurements taken on 72 standard bialkali 10-inch R7081 PMTs.

In order to measure the time and charge characteristics of the overall PMT response when the whole photocathode surface was uniformily illuminated, the PMT under test was positioned in a light-tight plastic dark box $(1 \times 0.5 \times 0.5 \mathrm{~m})$. The light source was a pulsed laser (PDL $800-\mathrm{B}$ produced by PICOQUANT) with a head of $410 \mathrm{~nm}$ and a width of $60 \mathrm{ps}$ as FWHM. It was located outside the box, and its light was split into two beams by an 80/20-ratio optical splitter. Using multimode optical fibres, the lower output was guided inside the test box, and the higher output was sent to a selected PMT positioned in a second dark box, which was used as a monitor of the laser source to generate a function for normalizing the various measurements.

To spread the laser beam in order to illuminate the whole photocathode surface, a calibrated optical diffuser was connected to the end of the optical fibre inside the test box. The PMT was positioned vertically, with its active area aligned perpendicularly to the axis of the 
fibre. The distance between the PMT head and the light source was set to $0.6 \mathrm{~m}$. In this condition the whole photocathode surface was illuminated. Figure 1 presents a schematic drawing of the apparatus.



Figure 1 Schematic view of the apparatus for illuminating the whole PMT

All measurements were carried out at room temperature and atmospheric pressure, on the PMTs powered by the same active base manufactured by ISEG, model PHQ7081 sel., with a partition ratio configured in accordance with Hamamatsu guidelines.

The performance of the tested PMTs was measured in response to a single photoelectron (s.p.e.) obtained by correctly attenuating the pulsed laser source. All the PMTs were tested at the same gain conditions of $5 \times 10^{7}$.

\subsection{Results}

The ratio between the number of the detected pulses on the number of the pulses emitted by the laser source defines the detector detection efficiency. In order to measure the improvement in quantum efficiency of the SBA PMTs as compared to the standards, comparative measurements of their detection efficiencies were made illuminating the whole photocathode surface of each PMT. Since the two families of PMTs have exactly the same mechanical design and internal structures, any increase in their detection efficiency can be attributed only to an improvement in their quantum efficiency.

Table 1 shows the quantum efficiency calculated for the SBA PMTs. As can be seen, the obtained results match with the $35 \%$ declared by Hamamatsu.

\begin{tabular}{l|c}
\hline \hline & QE calculated @ 410 nm [\%] \\
\hline SBA PMT 1 & 34.4 \\
\hline SBA PMT 2 & 33 \\
\hline SBA PMT 3 & 35 \\
\hline SBA PMT 4 & 34 \\
\hline \hline
\end{tabular}

Table 1 Quantum efficiency of the four SBA PMTs 
In order to define whether the quantum efficiency improvement process could introduce secondary effects in noise, and time and charge resolutions, the overall characteristics of the SBA PMTs were also measured. The dark count (DC) rate was measured with a threshold of $1 / 3$ of s.p.e. signal. The s.p.e. transit time spectrum was acquired for each PMT at the same threshold, and the transit time spread (TTS) was calculated as the FWHM of the histogram. Peak-to-valley ratio $(\mathrm{P} / \mathrm{V})$ and the sigma of the charge resolution (RES) were calculated from every acquired s.p.e. charge spectrum. Table 2 shows all the measurements, compared to the average values measured on the 72 standard (std.) PMTs [5].

\begin{tabular}{l|c|c|c|c|c}
\hline & $\begin{array}{l}\text { Volt @ gain } \\
\text { 5E7 }[\mathrm{V}]\end{array}$ & $\begin{array}{l}\text { DC rate } \\
{[\mathrm{Hz}]}\end{array}$ & P/V & $\begin{array}{l}\text { RES } \\
\text { sigma }[\%]\end{array}$ & $\begin{array}{l}\text { TTS } \\
\text { FWHM }[\mathrm{ns}]\end{array}$ \\
\hline 72 std. PMTs & $\mathbf{1 6 5 5}$ & $\mathbf{1 3 8 8}$ & $\mathbf{3 . 5}$ & $\mathbf{3 2}$ & $\mathbf{2 . 8}$ \\
\hline SBA PMT 1 & $\mathbf{1 7 6 0}$ & $\mathbf{4 8 0 5}$ & $\mathbf{2 . 8}$ & $\mathbf{2 7}$ & $\mathbf{2 . 8}$ \\
\hline SBA PMT 2 & $\mathbf{1 6 7 8}$ & $\mathbf{2 0 0 3}$ & $\mathbf{2 . 5}$ & $\mathbf{4 1}$ & $\mathbf{3 . 1}$ \\
\hline SBA PMT 3 & $\mathbf{1 7 6 0}$ & $\mathbf{3 2 6 5}$ & $\mathbf{3 . 0}$ & $\mathbf{3 3}$ & $\mathbf{2 . 7}$ \\
\hline SBA PMT 4 & $\mathbf{1 6 9 6}$ & $\mathbf{1 5 9 8}$ & $\mathbf{3 . 4}$ & $\mathbf{2 8}$ & $\mathbf{2 . 6}$ \\
\hline \hline
\end{tabular}

Table 2 Overall characteristics of the four SBA PMTs compared to those of 72 standard PMTs.

As can be seen in table 2, an increase in the dark count rate was measured for SBA PMTs with respect to the standards, when the measurements of charge and time resolutions were compatible with the average values found in standard PMTs.

Another measurement was the dark count rate decay time after a short exposure to light, measured for both standard and SBA photomultipliers. The tested PMTs were submitted without voltage to the ambient light of neon lamps for 5 seconds, and after that the dark count rate was measured as a function of the time from the instant of power on. Figure 2 shows the raw data. The results demonstrated that the SBA PMTs have a noise decay time of over $10 \mathrm{~h}$, greater than the $6 \mathrm{~h}$ measured on standard PMTs. This effect was also confirmed by other studies performed on SBA photomultipliers produced by other manufacturers [6].
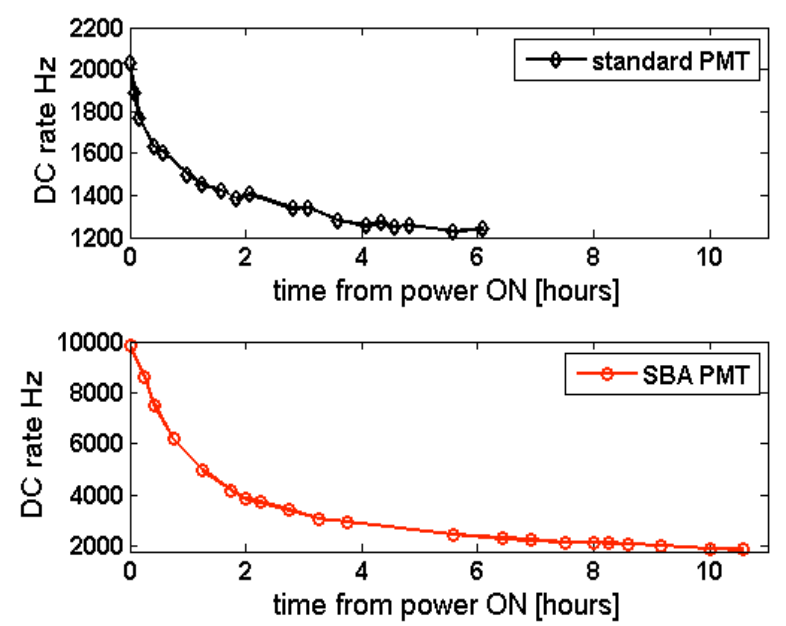

Figure 2 Dark count rate as function of time for standard and SBA photocathode PMTs 
Other important results were obtained measuring the fractions of the four different types of spurious pulses. Spurious pulses are noise pulses, time-correlated with the main PMT response. They are defined as:

- pre-pulses (P.P.), which appear 10-80 ns before the main pulses, and arise from a direct photo-effect on the first dynode, due to photons which pass the photocathode without interactions [7].

- late pulses (L.P.), which appear 10-80 ns after the main pulses, in place of it, and are caused by photoelectron backscattering on the first dynode [7].

- type 1 after pulse (AP1), which appears in the 10-80 ns interval time after the main pulse, and is due to luminous reaction on the electrodes bombarded by electrons.

- type 2 after pulse (AP2), which arrives in the 80 ns-16 $\mu$ s interval time after the main pulse, and is caused by the ionization of the residual gas in the PMT which is accelerated towards the photocathode.

For each of these four groups, the ratio of spurious pulses divided by the number of main pulses was measured with a threshold of $1 / 3$ of s.p.e for each PMT. Table 3 presents the measured fraction of spurious pulses, expressed as a percentage, and compared with the average values of the standard PMTs [5].

\begin{tabular}{l|c|c|c|c}
\hline \hline & P.P. $[\%]$ & L.P. $[\%]$ & AP1 $[\%]$ & AP2 $[\%]$ \\
\hline 72 std. PMT & 0.02 & 5.4 & 1.1 & 4.4 \\
\hline SBA PMT 1 & 0.03 & 5.8 & 1.9 & 15.2 \\
\hline SBA PMT 2 & 0.02 & 6.4 & 1.2 & 8.4 \\
\hline SBA PMT 3 & 0.01 & 5.7 & 1.6 & 11.3 \\
\hline SBA PMT 4 & 0.01 & 5.8 & 1.3 & 8.7 \\
\hline \hline
\end{tabular}

Table 3 Fractions of spurious pulses measured on the four SBA PMTs compared with the standards.

As can be seen, one considerable secondary effect of the SBA photocathode with respect to the standard one was an increase in the type 2 after pulse fraction. This effect is confirmed by studies on other SBA PMTs [8].

Since the occurrence time of the type 2 after pulse is closely correlated to the atomic mass of the residual gas, the afterpulse time distribution enables ions of different masses to be separated, so that the composition of the impurities inside the PMT can be studied [9]. With that in mind, the mean distributions of the delay-time of the type 2 after pulse from the main pulse were measured on the SBA PMTs, and were compared with the mean time distributions of standard PMTs. As shown in Figure 3, the arrival time distributions of type 2 after pulse were the same for the two families of PMTs, with a peak around $8 \mu$ s which could be produced by Cs ions and a peak around $2 \mu$ s which could be produced by $\mathrm{CH}_{4}$ ions [10]. This led to conclude that the improvement of the photocathode in the SBA PMTs does not introduce other type of ions, considering also that the fraction of events falling into the range of the two main peaks with respect to the total number of events remain the same for the standard and SBA PMTs. 



Figure 3 Distributions of the arrival time of the type 2 after pulses for SBA and standard PMTs

\section{Uniformity measurements}

Uniformity of a photomultiplier is commonly defined as the variation of the PMT response with respect to the photocathode incident position of the light spot [3]. Thus, for a large area photomultiplier, it is an important parameter. A comparison of local measurements of detection efficiency, made by scanning the photocathode surface was used to evaluate the uniformity of the SBA photocathode with respect to the standard. The laser source was attenuated to produce a single photoelectron pulsed laser beam collimated to obtain a $5 \mathrm{~mm}$-diameter which remained perpendicular to the PMT surface. The ratio of the number of s.p.e. pulses detected by the PMT under test to the number of pulses emitted by the laser was calculated for each measured point. The number of laser pulses emitted was simultaneously counted by the monitor PMT.

The results obtained confirmed that the uniformity of the SBA photocathode did not deteriorate with respect to that measured on standard bialkali. Figure 4 shows the detection efficiency measured along the vertical and horizontal axes.
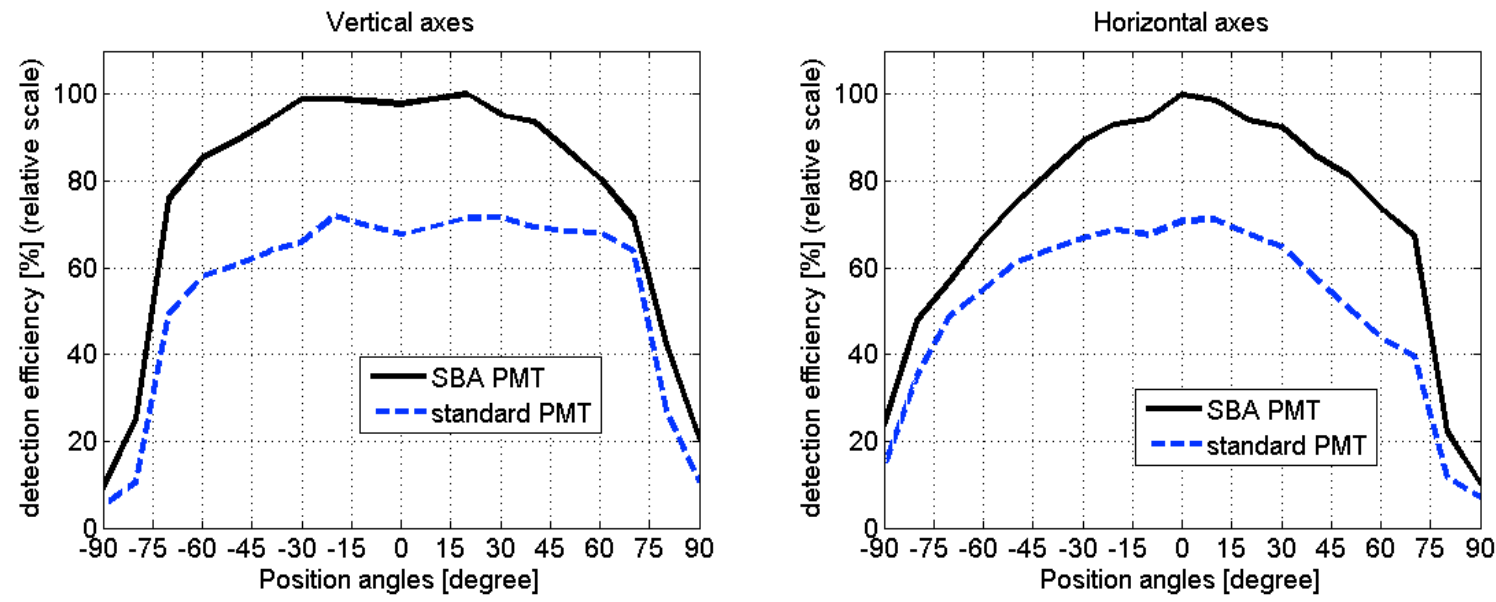

Figure 4 Detection efficiency along horizontal and vertical axes for a SBA and a standard PMT. 


\section{Ageing measurements}

In order to study whether the SBA photocathode could have differences about the change of the characteristics of the device during operating time, an accurate study about the ageing effect has been done on two 10 inch. R7081 PMT, one with a standard bialkali photocathode, and the other one with a super bialkali photocathode [11]. The measurement campaign, performed for almost three years, consisted in two different phases: accelerated ageing phase, and full characterization in single photo-electron condition. During the first phase, the photocathode of the two PMTs were illuminated with a 400nm LED regulated in order to produce pulsed signals of an average value of about 3 photo-electrons (pe) at a frequency of 1 $\mathrm{MHz}$. The ageing measurement of 3 years was then equivalent to about 45 years of operative life of a photomultiplier under a light source of 1 pe at $200 \mathrm{KHz}$. A 20/80 optical splitter was used to separate the beam in two parts: the upper part was connected to a bolometer to constantly measure the LED intensity. The lower part was connected to a calibrated 50/50 optical splitter, used to illuminate equally the two PMTs under test. Figure 5 shows the experimental set-up. The PMT power supply bases were two identical passive DC coupled bleeder following the scheme recommended by the manufacturer. The anode current of the two PMTs is continuously measured and acquired, together with the bolometer, by a calibrated DATA logger during the ageing phase.



Figure 5 Experimental set-up for the ageing measurements

Once a week the PMT ageing phase was stopped, the LED turned off and after a suitable reconditioning time the full characterization phase started. In this phase the pulsed laser source regulated in spe condition has been used to illuminate the whole photocathode surface and measuring gain, time and charge characteristics and fraction of spurious pulses. The measurements did not show differences between the behaviour of the two models of PMTs. The parameters of both the two photomultipliers haven't substantial changed during long operating time except for the gain. In Figure 6 the behaviour of the gain measured for both PMTs versus the total anode output is shown. First, an up-drift trend was measured, up to about $1500 \mathrm{C}$ and $2300 \mathrm{C}$ for the standard and the SBA PMT respectively, followed by a step decrease, 
corresponding to the down drift phase, until to the value of total charge collected of about 1800 $\mathrm{C}$ and $2500 \mathrm{C}$ for the standard and SBA PMT respectively.

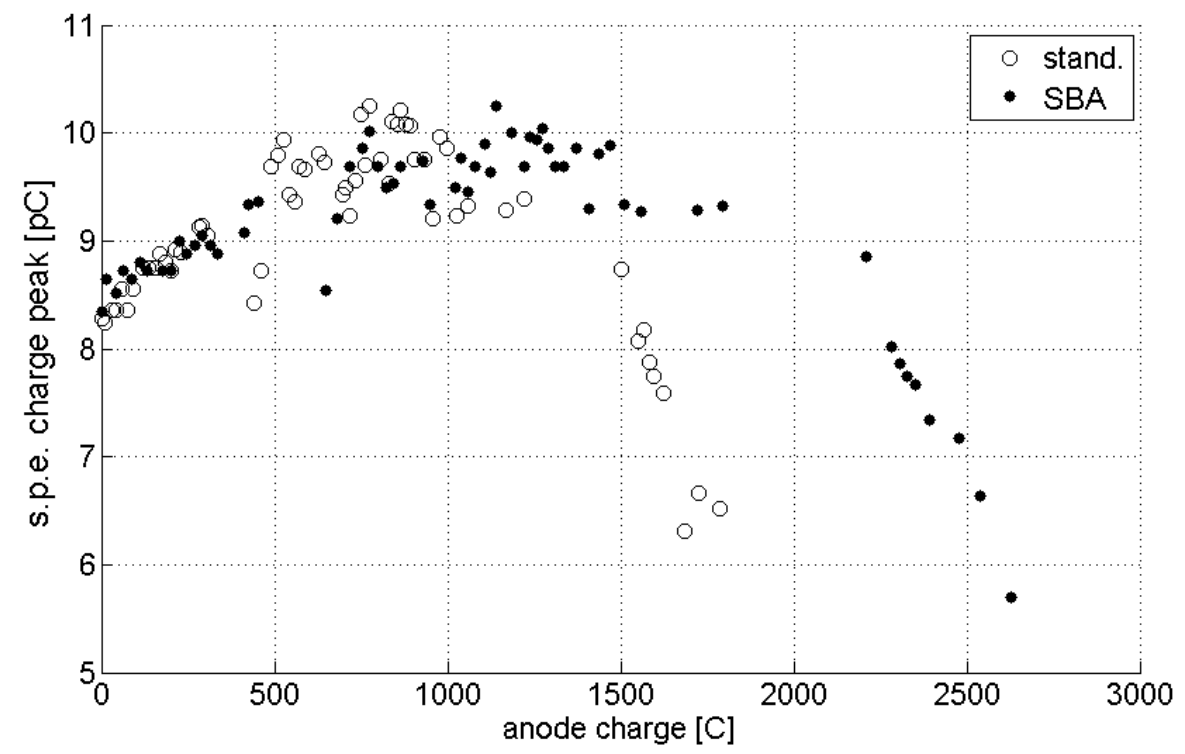

Figure 6 Trend of the gain during ageing measurements for the both types of PMTs

The up-drift phase showed an increase of the gain of about $10 \%$ and the following down drift phase showed a faster diminution of the gain of about $30 \%$. The ratio of the total charges measured in the same time is proportional to the ratio of the quantum efficiency of the two PMTs, as expected considering that they are illuminated by the same light intensity level. Moreover, measurements confirmed as the SBA PMT has about twice the type 2 after pulses fraction with respect to the standard PMT.

\section{Conclusion}

The performances of large area PMTs with super bialkali photocathode were measured and compared with those of standard bialkali PMTs. The most important result confirmed an increase in their quantum efficiency, with values which range in 33\%-35\% at the spectral peak, as declared by manufacturer. The time and charge resolutions measured on SBA PMTs were close to that of the standard PMT. A considerable increase was measured in noise pulses. The dark count rate was higher than standard, almost twice, and the fraction of type 2 after pulse was almost 3 times greater than that of the standard. The comparison of the time distribution of the type 2 after pulses confirmed as the SBA photocathode did not introduce other types of impurity inside the PMT with respect to the standard. A study with local measurements, made by scanning the photocathode surface, showed that the SBA photocathode uniformity was not worse than that of the standard bialkali photocathode. At last, an accurate study about the ageing effects has been performed for over three years, equivalent to about 45 years of operative life of a photomultiplier under a light source of 1 pe at $200 \mathrm{KHz}$, and it did not show differences between the two types of photocathode during the operating time. 


\section{References}

[1] J.A. Aguilar et al., Nucl. Instr. And Meth. A 555 (2005) 132-141

[2] K. Hanson et al., Nucl. Instr. And Meth. A 567 (2006) 214-217

[3] Photomultiplier Tube, principle to application. Hamamatsu, Japan, 1994

[4] Hamamatsu web site, < http://www.hamamatsu.com $>$

[5] S. Aiello, E. Leonora, et al. Nucl. Instr. Meth. A, 614 (2010) 206-212

[6] D. Dormic et al., Nucl. Instr. Meth. A 567 (2006) 27-30

[7] B. K.Lubsansorzhiev et al. Nucl. Instr. Meth. A 442 (2000) 452-458

[8] N. Akchurin, H.Kim, Nucl. Instr. Meth. A 574 (2007) 121-126

[9] P. B. Coates, J. Phys. D: Appl. Phys., Vol. 6 (1973)

[10] A. Tripathi at al., UCLA-Cosmic/2000-3

[11] S. Aiello et al., Nucl. Instr. and Meth. A (2012), DOI 10.1016/j.nima.2012.11.130 